\title{
Some Inequalities of Hermite-Hadamard Type for Functions Whose 3rd Derivatives Are P-Convex
}

\author{
Boyan $\mathrm{Xi}^{1}$, Shuhong Wang ${ }^{1}$, Feng $\mathbf{Q i}^{2}$ \\ ${ }^{1}$ College of Mathematics, Inner Mongolia University for Nationalities, Tongliao, China \\ ${ }^{2}$ School of Mathematics and Informatics, Henan Polytechnic University, Jiaozuo, China \\ Email: baoyintu78@qq.com, baoyintu68@sohu.com; shuhong7682@163.com, \\ qifeng618@gmail.com, qifeng618@hotmail.com, qifeng618@qq.com
}

Received September 30, 2012; revised October 30, 2012; accepted November 7, 2012

\begin{abstract}
In the paper, the authors establish some new Hermite-Hadamard type inequalities for functions whose 3rd derivatives are $P$-convex.
\end{abstract}

Keywords: Integral Inequality; Hermite-Hadamard's Integral Inequality; P-Convex Function; Derivative

\section{Introduction}

The following definition is well known in the literature.

Definition 1.1. A function $f: I \subset \mathbb{R} \rightarrow \mathbb{R}$ is said to be convex if

$$
\begin{aligned}
& f(\lambda x+(1-\lambda) y) \\
& \leq \lambda f(x)+(1-\lambda) f(y)
\end{aligned}
$$

holds for all $x, y \in I$ and $\lambda \in[0,1]$.

In [1], the concept of the so-called $P$-convex functions was introduced as follows.

Definition 1.2. ([1]) We say that a map

$f: I \subset \mathbb{R} \rightarrow \mathbb{R}$ belongs to the class $P(I)$ if it is nonnegative and satisfies

$$
f(\lambda x+(1-\lambda) y) \leq f(x)+f(y)
$$

for all $x, y \in I$ and $\lambda \in[0,1]$.

In [2], S. S. Dragomir proved the following theorems.

Theorem 1.1. ([2]) Let $f: I \subset \mathbb{R} \rightarrow \mathbb{R}$ be a differentiable mapping on $I^{\circ}$ and $a, b \in I^{\circ}$ with $a<b$. If $\left|f^{\prime}(x)\right|$ is convex on $[a, b]$, then

$$
\begin{aligned}
& \left|\frac{f(a)+f(b)}{2}-\frac{1}{b-a} \int_{a}^{b} f(x) \mathrm{d} x\right| \\
& \leq \frac{(b-a)\left(\left|f^{\prime}(a)\right|+\left|f^{\prime}(b)\right|\right)}{8},
\end{aligned}
$$

Theorem 1.2. ([2]) Let $f: I \subset \mathbb{R} \rightarrow \mathbb{R}$ be a differentiable mapping on $I^{\circ}$ and $a, b \in I^{\circ}$ with $a<b$. If $\left|f^{\prime}(x)\right|^{q}$ is convex on $[a, b]$ for $q \geq 1$, then

$$
\begin{aligned}
& \left|\frac{f(a)+f(b)}{2}-\frac{1}{b-a} \int_{a}^{b} f(x) \mathrm{d} x\right| \\
& \leq \frac{b-a}{4}\left(\frac{\left|f^{\prime}(a)\right|^{q}+\left|f^{\prime}(b)\right|^{q}}{2}\right)^{1 / q} .
\end{aligned}
$$

Theorem 1.3. ([3], Theorems 2) Let $f: I \subset \mathbb{R} \rightarrow \mathbb{R}$ be an absolutely continuous function on $I^{\circ}$ such that $f^{\prime \prime \prime} \in L([a, b])$ for $a, b \in I^{\circ}$ with $a<b$. If $\left|f^{\prime \prime \prime}(x)\right|$ is quasi-convex on $[a, b]$, then

$$
\begin{aligned}
& \left|\int_{a}^{b} f(x) \mathrm{d} x-\frac{b-a}{6}\left[f(a)+4 f\left(\frac{a+b}{2}\right)+f(b)\right]\right| \\
& \leq \frac{(b-a)^{4}}{1152}\left[\max \left\{\left|f^{\prime \prime \prime}(a)\right|, \mid f^{\prime \prime \prime}\left(\frac{a+b}{2}\right)\right\}\right\} \\
& \left.+\max \left\{\left|f^{\prime \prime \prime}\left(\frac{a+b}{2}\right)\right|,\left|f^{\prime \prime \prime}(b)\right|\right\}\right] .
\end{aligned}
$$

For more information and recent developments on this topic, please refer to [4-14] and closely related references therein.

The concepts of various convex functions have indeed found important places in contemporary mathematics as can be seen in a large number of research articles and books devoted to the field these days.

In this paper, we will establish some new HermiteHadamard type inequalities for functions whose 3 rd derivatives are $P$-convex. 


\section{A Lemma}

In this section, we establish an integral identity.
Lemma 2.1. Let $f: I \subset \mathbb{R} \rightarrow \mathbb{R}$ be a three times differentiable mapping on $I^{\circ}$ and $a, b \in I^{\circ}$ with $a<b$. If $f^{\prime} \in L[a, b]$, then

$$
\begin{aligned}
& \frac{1}{b-a} \int_{a}^{b} f(x) \mathrm{d} x-f\left(\frac{a+b}{2}\right)-\frac{(b-a)^{2}}{24} f^{\prime \prime}\left(\frac{a+b}{2}\right) \\
& =\frac{(b-a)^{3}}{96}\left\{\int_{0}^{1}(1-t)^{3} f^{\prime \prime \prime}\left(\frac{1-t}{2} a+\frac{1+t}{2} b\right) \mathrm{d} t-\int_{0}^{1}(1-t)^{3} f^{\prime \prime \prime}\left(\frac{1+t}{2} a+\frac{1-t}{2} b\right) \mathrm{d} t\right\} .
\end{aligned}
$$

Proof. Integrating by part and changing variable of definite integral yield

$$
\begin{aligned}
& \int_{0}^{1}(1-t)^{3} f^{\prime \prime \prime}\left(\frac{1+t}{2} a+\frac{1-t}{2} b\right) \mathrm{d} t=-\frac{2}{b-a} \int_{0}^{1}(1-t)^{3} \mathrm{~d} f^{\prime \prime}\left(\frac{1+t}{2} a+\frac{1-t}{2} b\right) \\
& =-\frac{2}{b-a}\left\{-f^{\prime \prime}\left(\frac{a+b}{2}\right)+3 \int_{0}^{1}(1-t)^{2} f^{\prime \prime}\left(\frac{1+t}{2} a+\frac{1-t}{2} b\right) \mathrm{d} t\right\} \\
& =\frac{2}{b-a} f^{\prime \prime}\left(\frac{a+b}{2}\right)+\frac{12}{(b-a)^{2}} \int_{0}^{1}(1-t)^{2} \mathrm{~d} f^{\prime}\left(\frac{1+t}{2} a+\frac{1-t}{2} b\right) \\
& =\frac{2}{b-a} f^{\prime \prime}\left(\frac{a+b}{2}\right)-\frac{12}{(b-a)^{2}} f^{\prime}\left(\frac{a+b}{2}\right)-\frac{48}{(b-a)^{3}} \int_{0}^{1}(1-t) \mathrm{d} f\left(\frac{1+t}{2} a+\frac{1-t}{2} b\right) \\
& =\frac{2}{b-a} f^{\prime \prime}\left(\frac{a+b}{2}\right)-\frac{12}{(b-a)^{2}} f^{\prime}\left(\frac{a+b}{2}\right) \\
& +\frac{48}{(b-a)^{3}}\left\{f\left(\frac{a+b}{2}\right)-\int_{0}^{1} f\left(\frac{1+t}{2} a+\frac{1-t}{2} b\right) \mathrm{d} t\right\}
\end{aligned}
$$

and

$$
\begin{aligned}
& \int_{0}^{1}(1-t)^{3} f^{\prime \prime \prime}\left(\frac{1-t}{2} a+\frac{1+t}{2} b\right) \mathrm{d} t=\frac{2}{b-a}(1-t) \int_{0}^{1}(1-t)^{3} \mathrm{~d} f^{\prime \prime}\left(\frac{1-t}{2} a+\frac{1+t}{2} b\right) \\
& =-\frac{2}{b-a} f^{\prime \prime}\left(\frac{a+b}{2}\right)+\frac{12}{(b-a)^{2}} \int_{0}^{1}(1-t)^{2} \mathrm{~d} f^{\prime}\left(\frac{1-t}{2} a+\frac{1+t}{2} b\right) \\
& =-\frac{2}{b-a} f^{\prime \prime}\left(\frac{a+b}{2}\right)-\frac{12}{(b-a)^{2}} f^{\prime}\left(\frac{a+b}{2}\right)+\frac{48}{(b-a)^{3}} \int_{0}^{1}(1-t) \mathrm{d} f\left(\frac{1-t}{2} a+\frac{1+t}{2} b\right) \\
& =-\frac{2}{b-a} f^{\prime \prime}\left(\frac{a+b}{2}\right)-\frac{12}{(b-a)^{2}} f^{\prime}\left(\frac{a+b}{2}\right)-\frac{48}{(b-a)^{3}}\left\{f\left(\frac{a+b}{2}\right)-\int_{0}^{1} f\left(\frac{1-t}{2} a+\frac{1+t}{2} b\right) \mathrm{d} t\right\} .
\end{aligned}
$$

The proof of Lemma 2.1 is complete.

\section{Hermite-Hadamard's Type Inequalities for $P$-Convex Functions}

Theorem 3.1. Let $f: I \subset \mathbb{R} \rightarrow \mathbb{R}$ be differentiable on $I^{\circ}, a, b \in I^{\circ}$ with $a<b$, and $f^{\prime \prime \prime} \in L([a, b])$. If $\left|f^{m^{\prime \prime}}\right|^{q}$ is $P$-convex on $[a, b]$ for $q \geq 1$, then

$$
\begin{aligned}
& \left|\frac{1}{b-a} \int_{a}^{b} f(x) \mathrm{d} x-f\left(\frac{a+b}{2}\right)-\frac{(b-a)^{2}}{24} f^{\prime \prime}\left(\frac{a+b}{2}\right)\right| \\
& \leq \frac{(b-a)^{3}}{192}\left[\left|f^{\prime \prime \prime}(a)\right|^{q}+\left|f^{\prime \prime \prime}(b)\right|^{q}\right]^{1 / a} .
\end{aligned}
$$

Proof. Since $\left|f^{\prime \prime \prime}\right|^{q}$ is a $P$-convex function on $[a, b]$, by Lemma 2.1 and Hölder's inequality, we obtain 


$$
\begin{aligned}
& \left|\frac{1}{b-a} \int_{a}^{b} f(x) \mathrm{d} x-f\left(\frac{a+b}{2}\right)-\frac{(b-a)^{2}}{24} f^{\prime \prime}\left(\frac{a+b}{2}\right)\right| \leq \frac{(b-a)^{3}}{96}\left\{\int_{0}^{1}(1-t)^{3}\left|f^{\prime \prime \prime}\left(\frac{1-t}{2} a+\frac{1+t}{2} b\right)\right| \mathrm{d} t\right. \\
& \left.+\int_{0}^{1}(1-t)^{3}\left|f^{\prime \prime \prime}\left(\frac{1+t}{2} a+\frac{1-t}{2} b\right)\right| \mathrm{d} t\right\} \leq \frac{(b-a)^{3}}{96}\left(\int_{0}^{1}(1-t)^{3} \mathrm{~d} t\right)^{1-1 / q} \\
& \times\left\{\left(\int_{0}^{1}(1-t)^{3}\left|f^{\prime \prime \prime}\left(\frac{1-t}{2} a+\frac{1+t}{2} b\right)\right|^{q} \mathrm{~d} t\right)^{1 / q}+\left(\int_{0}^{1}(1-t)^{3}\left|f^{\prime \prime \prime}\left(\frac{1+t}{2} a+\frac{1-t}{2} b\right)\right|^{q} \mathrm{~d} t\right)^{1 / q}\right\} \\
& \leq \frac{(b-a)^{3}}{48}\left(\int_{0}^{1}(1-t)^{3} \mathrm{~d} t\right)^{1-1 / q}\left(\int_{0}^{1}(1-t)^{3} \mathrm{~d} t\right)^{1 / q}\left[\left|f^{\prime \prime \prime}(a)\right|^{q}+\left|f^{\prime \prime \prime}(b)\right|^{q}\right]^{1 / q} \\
& =\frac{(b-a)^{3}}{48}\left(\int_{0}^{1}(1-t)^{3} \mathrm{~d} t\right)\left[\left|f^{\prime \prime \prime}(a)\right|^{q}+\left|f^{\prime \prime \prime}(b)\right|^{q}\right]^{1 / q}=\frac{(b-a)^{3}}{192}\left[\left|f^{\prime \prime \prime}(a)\right|^{q}+\left|f^{\prime \prime \prime}(b)\right|^{q}\right]^{1 / q} .
\end{aligned}
$$

The proof of Theorem 3.1 is complete.

Corollary 3.1.1. Under the conditions of Theorem 3.1, if $q=1$, we have

$$
\left|\frac{1}{b-a} \int_{a}^{b} f(x) \mathrm{d} x-f\left(\frac{a+b}{2}\right)-\frac{(b-a)^{2}}{24} f^{\prime \prime}\left(\frac{a+b}{2}\right)\right| \leq \frac{(b-a)^{3}}{192}\left[\left|f^{\prime \prime \prime}(a)\right|+\left|f^{\prime \prime \prime}(b)\right|\right] .
$$

Theorem 3.2. Let $f: I \subset \mathbb{R} \rightarrow \mathbb{R}$ be differentiable on $I^{\circ}, a, b \in I^{\circ}$ with $a<b$, and $f^{\prime \prime \prime} \in L([a, b])$. If $\left|f^{\prime \prime \prime}\right|^{q}$ is $P$-convex on $[a, b]$ for $q>1$, then

$$
\left|\frac{1}{b-a} \int_{a}^{b} f(x) \mathrm{d} x-f\left(\frac{a+b}{2}\right)-\frac{(b-a)^{2}}{24} f^{\prime \prime}\left(\frac{a+b}{2}\right)\right| \leq \frac{(b-a)^{3}}{48}\left(\frac{1}{3 q+1}\right)^{1 / a}\left[\left|f^{\prime \prime \prime}(a)\right|^{q}+\left|f^{\prime \prime \prime}(b)\right|^{q}\right]^{1 / a} .
$$

Proof. From Lemma 2.1, Hölder's inequality, and the $P$-convexity of $\left|f^{\prime \prime \prime}\right|^{q}$ on $[a, b]$, we drive

$$
\begin{aligned}
& \left|\frac{1}{b-a} \int_{a}^{b} f(x) \mathrm{d} x-f\left(\frac{a+b}{2}\right)-\frac{(b-a)^{2}}{24} f^{\prime \prime}\left(\frac{a+b}{2}\right)\right| \leq \frac{(b-a)^{3}}{96}\left\{\int_{0}^{1}(1-t)^{3}\left|f^{\prime \prime \prime}\left(\frac{1-t}{2} a+\frac{1+t}{2} b\right)\right| \mathrm{d} t\right. \\
& \left.+\int_{0}^{1}(1-t)^{3}\left|f^{\prime \prime \prime}\left(\frac{1+t}{2} a+\frac{1-t}{2} b\right)\right| \mathrm{d} t\right\} \leq \frac{(b-a)^{3}}{96}\left(\int_{0}^{1} 1 \mathrm{~d} t\right)^{1-1 / q} \\
& \times\left\{\left(\left.\int_{0}^{1}(1-t)^{3 a}\left|f^{\prime \prime \prime}\left(\frac{1-t}{2} a+\frac{1+t}{2} b\right)\right|\right|^{q} \mathrm{~d} t\right)^{1 / q}+\left(\int_{0}^{1}(1-t)^{3 q}\left|f^{\prime \prime \prime}\left(\frac{1+t}{2} a+\frac{1-t}{2} b\right)\right|^{q} \mathrm{~d} t\right)^{1 / q}\right\} \\
& \leq \frac{(b-a)^{3}}{48}\left(\int_{0}^{1}(1-t)^{3 q} \mathrm{~d} t\right)^{1 / q}\left[\left|f^{\prime \prime \prime}(a)\right|^{q}+\left|f^{\prime \prime \prime}(b)\right|^{q}\right]^{1 / q}=\frac{(b-a)^{3}}{48}\left(\frac{1}{3 q+1}\right)^{1 / q}\left[\left|f^{\prime \prime \prime}(a)\right|^{q}+\left|f^{\prime \prime \prime}(b)\right|^{q}\right]^{1 / q} .
\end{aligned}
$$

Theorem 3.2 is proved.

Theorem 3.3. Let $f: I \subset \mathbb{R} \rightarrow \mathbb{R}$ be differentiable on $I^{\circ}, a, b \in I^{\circ}$ with $a<b$, and $f^{\prime \prime \prime} \in L([a, b])$. If $\left|f^{\prime \prime \prime}\right|^{q}$ is $P$-convex on $[a, b]$ for $q>1$, then

$$
\left|\frac{1}{b-a} \int_{a}^{b} f(x) \mathrm{d} x-f\left(\frac{a+b}{2}\right)-\frac{(b-a)^{2}}{24} f^{\prime \prime}\left(\frac{a+b}{2}\right)\right| \leq \frac{(b-a)^{3}}{48}\left(\frac{q-1}{4 q-1}\right)^{1 / q}\left[\left|f^{\prime \prime \prime}(a)\right|^{q}+\left|f^{\prime \prime \prime}(b)\right|^{q}\right]^{1 / q} .
$$

Proof. From Lemma 2.1, Hölder's inequality, and the $P$-convexity of $\left|f^{\prime \prime \prime}\right|^{q}$ on $[a, b]$, we have 


$$
\begin{aligned}
& \left|\frac{1}{b-a} \int_{a}^{b} f(x) \mathrm{d} x-f\left(\frac{a+b}{2}\right)-\frac{(b-a)^{2}}{24} f^{\prime \prime}\left(\frac{a+b}{2}\right)\right| \leq \frac{(b-a)^{3}}{96}\left\{\int_{0}^{1}(1-t)^{3}\left|f^{\prime \prime \prime}\left(\frac{1-t}{2} a+\frac{1+t}{2} b\right)\right| \mathrm{d} t\right. \\
& \left.+\int_{0}^{1}(1-t)^{3}\left|f^{\prime \prime \prime}\left(\frac{1+t}{2} a+\frac{1-t}{2} b\right)\right| \mathrm{d} t\right\} \leq \frac{(b-a)^{3}}{96}\left(\int_{0}^{1}(1-t)^{3 q /(q-1)} \mathrm{d} t\right)^{1-1 / q} \\
& \times\left\{\left(\int_{0}^{1}\left|f^{\prime \prime \prime}\left(\frac{1-t}{2} a+\frac{1+t}{2} b\right)\right|^{q} \mathrm{~d} t\right)^{1 / q}+\left(\left.\int_{0}^{1}\left|f^{\prime \prime \prime}\left(\frac{1+t}{2} a+\frac{1-t}{2} b\right)\right|\right|^{q} \mathrm{~d} t\right)^{1 / q}\right\} \\
& \leq \frac{(b-a)^{3}}{48}\left(\int_{0}^{1}(1-t)^{3 a /(q-1)} \mathrm{d} t\right)^{1-1 / q}\left[\left|f^{\prime \prime \prime}(a)\right|^{q}+\left|f^{\prime \prime \prime}(b)\right|^{q}\right]^{1 / q}=\frac{(b-a)^{3}}{48}\left(\frac{q-1}{4 q-1}\right)^{1 / q}\left[\left|f^{\prime \prime \prime}(a)\right|^{q}+\left|f^{\prime \prime \prime}(b)\right|^{q}\right]^{1 / q} .
\end{aligned}
$$

Theorem 3.3 is thus proved.

Theorem 3.4. Let $f: I \subset \mathbb{R} \rightarrow \mathbb{R}$ be differentiable on $I^{\circ}, a, b \in I^{\circ}$ with $a<b$, and $f^{\prime \prime \prime} \in L([a, b])$. If $\left|f^{\prime \prime \prime}\right|^{q}$ for $q>1$ is $P$-convex on $[a, b]$ and $0<r, s<3$, then

$$
\begin{aligned}
& \left|\frac{1}{b-a} \int_{a}^{b} f(x) \mathrm{d} x-f\left(\frac{a+b}{2}\right)-\frac{(b-a)^{2}}{24} f^{\prime \prime}\left(\frac{a+b}{2}\right)\right| \\
& \leq \frac{(b-a)^{3}}{96}\left\{\left(\frac{q-1}{(4-s) q-1}\right)^{1-1 / q}\left(\frac{1}{s q+1}\right)^{1 / q}+\left(\frac{q-1}{(4-r) q-1}\right)^{1-1 / q}\left(\frac{1}{r q+1}\right)^{1 / q}\right\}\left[\left|f^{\prime \prime \prime}(a)\right|^{q}+\left|f^{\prime \prime \prime}(b)\right|^{q}\right]^{1 / q} .
\end{aligned}
$$

Proof. Using Lemma 2.1, Hölder's inequality, and the $P$-convexity of $\left|f^{\prime \prime \prime}\right|^{q}$ on $[a, b]$ yields

$$
\begin{aligned}
& \left|\frac{1}{b-a} \int_{a}^{b} f(x) \mathrm{d} x-f\left(\frac{a+b}{2}\right)-\frac{(b-a)^{2}}{24} f^{\prime \prime}\left(\frac{a+b}{2}\right)\right| \leq \frac{(b-a)^{3}}{96}\left\{\int_{0}^{1}(1-t)^{3}\left|f^{\prime \prime \prime}\left(\frac{1-t}{2} a+\frac{1+t}{2} b\right)\right| \mathrm{d} t\right. \\
& \left.+\int_{0}^{1}(1-t)^{3}\left|f^{\prime \prime \prime}\left(\frac{1+t}{2} a+\frac{1-t}{2} b\right)\right| \mathrm{d} t\right\} \leq \frac{(b-a)^{3}}{96}\left\{\left(\int_{0}^{1}(1-t)^{(3-s) q /(q-1)} \mathrm{d} t\right)^{1-1 / q}\right. \\
& \left.\times\left(\int_{0}^{1}(1-t)^{s q}\left|f^{\prime \prime \prime}\left(\frac{1-t}{2} a+\frac{1+t}{2} b\right)\right|{ }^{q} \mathrm{~d} t\right)^{1 / q}+\left(\int_{0}^{1}(1-t)^{(3-r) q /(q-1)} \mathrm{d} t\right)^{1-1 / q}\left(\int_{0}^{1}(1-t)^{r q}\left|f^{\prime \prime \prime}\left(\frac{1+t}{2} a+\frac{1-t}{2} b\right)\right|^{q} \mathrm{~d} t\right)^{1 / q}\right\} \\
& \leq \frac{(b-a)^{3}}{96}\left\{\left(\int_{0}^{1}(1-t)^{(3-s) q /(q-1)} \mathrm{d} t\right)^{1-1 / q}\left(\int_{0}^{1}(1-t)^{s q} \mathrm{~d} t\right)^{1 / q}+\left(\int_{0}^{1}(1-t)^{(3-r) q /(q-1)} \mathrm{d} t\right)^{1-1 / q}\left(\int_{0}^{1}(1-t)^{r q} \mathrm{~d} t\right)^{1 / q}\right\} \\
& \times\left[\left|f^{\prime \prime \prime}(a)\right|^{q}+\left|f^{\prime \prime \prime}(b)\right|^{q}\right]^{1 / q} \\
& =\frac{(b-a)^{3}}{96}\left\{\left(\frac{q-1}{(4-s) q-1}\right)^{1-1 / q}\left(\frac{1}{s q+1}\right)^{1 / q}+\left(\frac{q-1}{(4-r) q-1}\right)^{1-1 / q}\left(\frac{1}{r q+1}\right)^{1 / q}\right\}\left[\left|f^{\prime \prime \prime}(a)\right|^{q}+\left|f^{\prime \prime \prime}(b)\right|^{q}\right]^{1 / q} .
\end{aligned}
$$

The proof of Theorem 3.4 is complete.

Corollary 3.3.1. Under the conditions of Theorem 3.4,

(1) if $r=s$, then

$\left|\frac{1}{b-a} \int_{a}^{b} f(x) \mathrm{d} x-f\left(\frac{a+b}{2}\right)-\frac{(b-a)^{2}}{24} f^{\prime \prime}\left(\frac{a+b}{2}\right)\right| \leq \frac{(b-a)^{3}}{48}\left(\frac{q-1}{(4-r) q-1}\right)^{1-1 / q}\left(\frac{1}{r q+1}\right)^{1 / q} \times\left[\left|f^{\prime \prime \prime}(a)\right|^{q}+\left|f^{\prime \prime \prime}(b)\right|^{q}\right]^{1 / q} ;$

(2) if $r=s=1$, then 


$$
\left|\frac{1}{b-a} \int_{a}^{b} f(x) \mathrm{d} x-f\left(\frac{a+b}{2}\right)-\frac{(b-a)^{2}}{24} f^{\prime \prime}\left(\frac{a+b}{2}\right)\right| \leq \frac{(b-a)^{3}}{48}\left(\frac{q-1}{3 q-1}\right)^{1-1 / q}\left(\frac{1}{q+1}\right)^{1 / q}\left[\left|f^{\prime \prime \prime}(a)\right|^{q}+\left|f^{\prime \prime \prime}(b)\right|^{q}\right]^{1 / q}
$$

(3) if $r=s=2$, then

$$
\left|\frac{1}{b-a} \int_{a}^{b} f(x) \mathrm{d} x-f\left(\frac{a+b}{2}\right)-\frac{(b-a)^{2}}{24} f^{\prime \prime}\left(\frac{a+b}{2}\right)\right| \leq \frac{(b-a)^{3}}{48}\left(\frac{q-1}{2 q-1}\right)^{1-1 / q}\left(\frac{1}{2 q+1}\right)^{1 / q}\left[\left|f^{\prime \prime \prime}(a)\right|^{q}+\left|f^{\prime \prime \prime}(b)\right|^{q}\right]^{1 / q} .
$$

Finally we would like to note that these Hermite-Hadamard type inequalities obtained in this paper can be applied to the fields of integral inequalities, approximation theory, special means theory, optimization theory, information theory, and numerical analysis, as done before by a number of mathematicians.

\section{Acknowledgements}

The first two authors were partially supported by the Science Research Funding of Inner Mongolia University for Nationalities under Grant No. NMD1103.

\section{REFERENCES}

[1] S. S. Dragomir, J. Pečarić and L. E. Persson, "Some Inequalities of Hadamard Type," Soochow Journal of Mathematics, Vol. 21, No. 3, 1995, pp. 335-341.

[2] S. S. Dragomir and R. P. Agarwal, "Two Inequalities for Differentiable Mappings and Applications to Special Means of Real Numbers and to Trapezoidal Formula," Applied Mathematics Letters, Vol. 11, No. 5, 1998, pp. 91-95. doi:10.1016/S0893-9659(98)00086-X

[3] M. Alomari and S. Hussain, Two Inequalities of Simpson Type for Quasi-Convex Functions and Applications, Applied Mathematics E-Notes, Vol. 11, 2011, pp. 110-117.

[4] L. Chun and F. Qi, "Integral Inequalities of Hermite-Hadamard Type for Functions Whose 3rd Derivatives Are s-Convex," Applied Mathematics, Vol. 3, No. 11, 2012, pp. 1680-1685. doi:10.4236/am.2012.311232

[5] S. S. Dragomir and C. E. M. Pearce, "Selected Topics on Hermite-Hadamard Type Inequalities and Applications," RGMIA Monographs, Victoria University, Melbourne, 2000.

[6] W.-D. Jiang, D.-W. Niu, Y. Hua, and F. Qi, "Generalizations of Hermite-Hadamard Inequality to $n$-Time Differentiable Functions Which Are s-Convex in the Second
Sense," Analysis (Munich), Vol. 32, No. 3, 2012, pp. 209-220. doi:10.1524/anly.2012.1161

[7] U. S. Kirmaci, "Inequalities for Differentiable Mappings and Applications to Special Means of Real Numbers to Midpoint Formula," Applied Mathematics and Computation, Vol. 147, No. 1, 2004, pp. 137-146. doi:10.1016/S0096-3003(02)00657-4

[8] C. P. Niculescu and L.-E. Persson, "Convex Functions and Their Applications," Springer-Verlag, New York, 2005.

[9] C. E. M. Pearce and J. Pečarić, "Inequalities for Differentiable Mappings with Application to Special Means and Quadrature Formulae," Applied Mathematics Letters, Vol. 13, No. 2, 2000, pp. 51-55. doi:10.1016/S0893-9659(99)00164-0

[10] F. Qi, Z.-L. Wei and Q. Yang, "Generalizations and Refinements of Hermite-Hadamard's Inequality," Rocky Mountain Journal of Mathematics, Vol. 35, No. 1, 2005, pp. 235-251. doi:10.1216/rmjm/1181069779

[11] S.-H. Wang, B.-Y. Xi and F. Qi, "Some New Inequalities of Hermite-Hadamard Type for $n$-Time Differentiable Functions Which Are m-Convex," Analysis (Munich), Vol. 32, No. 3, 2012, pp. 247-262. doi:10.1524/anly.2012.1167

[12] B.-Y. Xi, R.-F. Bai and F. Qi, "Hermite-Hadamard Type Inequalities for the $m$-and $(a, m)$-Geometrically Convex Functions," Aequationes Mathematicae, Vol. 84, No. 3, 2012, pp. 261-269. doi:10.1007/s00010-011-0114-x

[13] B.-Y. Xi and F. Qi, "Some Integral Inequalities of Hermite-Hadamard Type for Convex Functions with Applications to Means," Journal of Function Spaces and Applications, Vol. 2012, 2012, Article ID: 980438, p 14. doi: $10.1155 / 2012 / 980438$

[14] T.-Y. Zhang, A.-P. Ji and F. Qi, “On Integral Inequalities of Hermite-Hadamard Type for s-Geometrically Convex Functions," Abstract and Applied Analysis, Vol. 2012, 2012, Article ID: 560586, p 14. doi:10.1155/2012/560586 